\title{
El Aprendizaje Significativo de las Ciencias Morfológicas en Medicina: Experiencia y Aportes para su Enseñanza en Clínica Dermatológica
}

\author{
The Significant Learning of Morphological Medicine Sciences: Experience \\ and Contributions for its Education in Dermatological Clinic
}

"María Elisa Dionisio de Cabalier \& **Delia María Chalub

DiONiSiO DE CABALIER, M. E. \& CHALUB, D. M. El aprendizaje significativo de las ciencias morfológicas en medicina: experiencia y aportes para su enseñanza en clínica dermatológica. Int. J. Morphol., 27(2):565-569, 2009.

RESUMEN: El trabajo es un análisis de la capacidad de los alumnos para reconocer significativamente con conceptos claros y precisos, distintas manifestaciones de los tejidos y patologías celulares en el campo de la Dermatología teniendo en cuenta los aportes de las ciencias morfológicas para conocer estructural y funcionalmente la unidad básica de la constitución del organismo humano: las células y los tejidos. En la propuesta de enseñanza y aprendizaje, tanto como en la evaluación sobre la cual se ha realizado la experiencia, se sostiene que el aprendizaje de estos conocimientos se requiere no sólo de habilidades, sino fundamentalmente de la capacidad para otorgar "sentido" o "significatividad", entre los conocimientos previos y los nuevos, entre los teóricos y los prácticos, entre la comprensión y las manifestaciones clínicas de los tejidos. En este trabajo se sostiene que la formación teórica tiene un papel muy importante en el aprendizaje de la morfología humana, en la medida que el aprendizaje de cada noción o concepto, es previa y básica para la realización de procedimientos y el desarrollo de habilidades. Los hallazgos demuestran que la significación integral de las patologías dermatológicas, es más dificultosa que la identificación de las células y los tejidos, pudiendo hipotetizar sobre la falta de integración cognitiva de los aprendizajes de los alumnos. Finalmente, se ofrecen algunas líneas de acción pedagógica basada en el mismo modelo educacional.

PAlabraS ClaVE: Aprendizaje significativo; Sentido; Comprensión; Razonamiento clínico; Reconocimiento; Histofisiopatologías dermatológicas.

\section{INTRODUCCIÓN}

En materia de educación médica, muchos trabajos se publicaron relatando nuevas experiencias de enseñanza y aprendizaje. No obstante, dado que Medicina no es un campo científico unitario, sino esencialmente interdisciplinario, hemos sentido la necesidad de realizar un aporte pedagógico vinculado al aprendizaje de las ciencias morfológicas en Medicina.

Con concepciones epistemológicas y pedagógicas que a continuación se exponen, se realizó un análisis de la capacidad de los alumnos de la cátedra de Dermatología de la Escuela de Medicina de la Universidad Nacional de Córdoba, para reconocer significativamente con conceptos científicos claros y precisos, distintas manifestaciones de las patologías en piel. Desde el punto de vista epistemológico, partimos de concebir a la Medicina, como ciencia conformada por conceptos teóricos, destrezas y procedimientos que requieren del dominio de recursos de tecnología médica específica; destinadas a diagnosticar, tratar y prevenir enfermedades. Dentro de esta amplia definición, las Ciencias Morfológicas se constituyen en el fundamento del conocimiento médico en la medida que es necesario conocer cómo está constituido el organismo humano - tanto en los aspectos estructurales como en los funcionales-, para reconocer después las distintas patologías de un órgano o sistema, a partir de la relación entre lo normal y las manifestaciones patológicas.

En el campo de la Medicina, la Morfología Humana es un campo de conocimiento interdisciplinario que se nutre

\footnotetext{
${ }^{*}$ Responsable de C.O.N.E.A.U. Secretaría de Graduados U.N.C, Córdoba, Argentina.

**Asesora Pedagógica de C.O.N.E.A.U. Secretaría de Graduados U.N.C. Córdoba, Argentina.
} 
de los aportes de: la Biología Molecular, la Anatomía, la Fisiología, la Histología, la Embriología y la Genética; para el estudio de los tejido y sus funciones en vistas a la caracterización integral del organismo humano (sobre el carácter interdisciplinario del campo de las ciencias morfológicas y sus consecuencias para la enseñanza, ha se había expresado en 1976 el «Informe del Comité del Programa de Libros de Texto para la OPS/OMS». En las recomendaciones se solicitaba relacionar las ciencias morfológicas entre sí y éstas con otras disciplinas de la salud, dentro de un proceso amplio y totalizador.

La Morfología se ocupa de la unidad constitutiva del ser humano en la medida que el fundamento celular su correcto o mal funcionamiento-, está implicados en la manifestación de una patología. Por lo tanto, el aprendizaje y el conocimiento de las Ciencias Morfológicas, requiere no sólo la asimilación de conceptos o, en el otro extremo, del dominio de habilidades; sino fundamentalmente la capacidad de otorgar sentido, establecer relaciones entre conocimientos y desarrollar prácticas orientadas por la comprensión significativa entre los conocimientos teóricos y las evidencias prácticas de la morfología celular. El valor de las Ciencias Morfológicas en la formación médica, radica en los conocimientos sobre los cuales los alumnos puedan generar prácticas acordes en base a la significatividad o el sentido, que el estudiante y futuro profesional, puedan dar a aquello que "nombra, reconoce o describe" en los estudios específicos o complementarios que revelan la situación clínica de un paciente.

Desde el punto de vista de los fundamentos pedagógicos en este trabajo, sostuvimos siempre que la formación teórica tiene un papel muy importante en el aprendizaje de la morfología humana (Jones et al., 2001), en la medida que el aprendizaje de cada noción o concepto, es previa y básica para la realización de procedimientos y el desarrollo de habilidades. Así, cuando los conocimientos teóricos han sido aprendidos significativamente, éstos se incorporan en la estructura cognitiva de los estudiantes y se constituyen en el fundamento para el desempeño hábil de una actividad clínica.

En este trabajo definimos al aprendizaje significativo desde el modelo psicológico y epistemológico cognitivo desarrollado por David Ausubel en colaboración con Novak, hacia la década del 70 con el propósito de explicar cómo operan las estructuras de pensamiento mediante esquemas de formación diversos (conformados por enfoques, teorías, conceptos y sus relaciones) al otorgar sentido a la realidad $\mathrm{y}$, en consecuencia, a las prácticas o cualquier tipo de acción-operatoria: formal o concreta-, sobre un campo objeto de estudio.
El proceso de significación, implica la capacidad para entablar relaciones entre los conocimientos teóricos y los prácticos, los conocimientos previos y los nuevos, entre los conocimientos más generales y abstractos y los más específicos -concretos y empíricos- en los que se contienen los de mayor nivel de generalidad (Zabala, 2003); en la estructura cognitiva del sujeto que aprende, es decir, desde sus formas de pensamiento entendiendo por tal, a toda operación sobre el mundo y las situaciones problemáticas que se le plantean al estudiante y futuro profesional. En este sentido aprender es un proceso que se asimila al de pensar en la medida que implica partir de lo que el sujeto ya sabe -conocimientos previos- y aplicar estrategias específicas sobre una situación que se me plantea como un problema a interpretar e intervenir (Ausubel et al., 2000).

Para que cada concepto pueda ser aprendido significativamente, el estudiante debe ser capaz de entender o comprender el significado. En términos del propio David Ausubel, algo tiene significado para alguien, cuando logra entenderlo y darle sentido", el autor agrega que "un aprendizaje es útil cuando la persona que lo ha realizado puede ponerlo en práctica para resolver problemas" en el que está imbuido un término. Un alumno comprende el significado de los términos teóricos cuando puede definirlo, explicarlo y en el caso de la especificidad de la Medicina, reconocer el concepto en los indicios clínicos de un paciente o los datos reveladores de los estudios micro y macroscópicos realizados. Sólo con una «asimilación comprensiva» (Pérez \& Diez, 2001) de los conocimientos de la Morfología Humana, podrá luego el estudiante ser capaz de reconocer las etiofisiopatologías que afecten al organismo de un paciente concreto.

La significación de cada concepto, a su vez, deviene de su relación con otros que conforman los tejidos humanos dada la naturaleza interdisciplinaria de las ciencias Morfológicas. La interrelación significativa de los conocimientos propios de la Morfología Humana, requiere del análisis dinámico de su origen y evolución; por esta razón, hemos sostenido que la posibilidad de generar aprendizajes significativos sobre el campo de estudio de estas ciencias, depende en gran parte del trabajo docente para generar en el alumno, formas inclusivas de interpretación (Rosell \& Puig, 2004).

El aporte de este trabajo, además de los hallazgos de la experiencia, atiene como propósito contribuir al pensamiento teórico sobre la pedagogía médica para desarrollar sistemas de enseñanza que tengan como último fin formar a un estudiante con saberes internalizados de forma comprensiva para analizar desde esquemas de pensamiento sobre morfología, sus prácticas en torno a los pacientes y la misma investigación en Medicina. 


\section{MATERIAL Y MÉTODO}

Este trabajo se sustenta en la metodología de enseñanza y aprendizaje significativo sostenida desde lo expuesto en el punto anterior. Desde este enfoque, los docentes de la cátedra de Clínica Dermatológica del cuarto año de la carrera de grado en Medicina de la Universidad Nacional de Córdoba, desarrollamos durante el año lectivo una propuesta de enseñanza de la Morfología Humana sustentada en el enfoque del aprendizaje significativo. El programa de contenidos de la asignatura, se desarrolla de menor a mayor nivel de complejidad, incluyendo de manera paulatina los conceptos de: células, tejidos, anatomía y patologías de los mismos. De esta forma, desde una visión recursiva (con la noción de recursividad, lo que hacemos es poner énfasis en el aprendizaje como proceso que no lineal, progresivo y acumulativo). Al contrario, requiere de operaciones mentales como analizar la nueva información, comprenderla, aclarar, verificar, experimentar. Este proceso es el propio del espíritu de investigación científica en el cual el pensamiento "hipotético" es esencial para analizar e interpretar fenómenos o hechos. A su vez es esencial en el razonamiento clínico en la medida que es la médula de la práctica médica en la medida que implica la capacidad de observación y reflexión del médico, la capacidad de elaborar un juicio sobre el paciente y sus patologías. Ya Hipócrates pone en relieve algunas características fundamentales del razonamiento clínico: el imprescindible recurso de la experiencia y su consecuente inseguridad, así como la dificultad del juicio. Baste para ilustrar este punto recordar todo lo que la crítica proveniente del interior de la propia práctica clínica ha señalado recientemente en cuanto a lo que a estas dificultades se refiere, principalmente a la tendencia común entre los médicos a configurar sus hipótesis diagnósticas y las configuraciones mismas de los síntomas y los diagnósticos de acuerdo a su propio conocimiento y opiniones. No faltan los autores que se admiran de que un buen, porcentaje de los juicios clínicos quedan establecidos antes de haberse obtenido del paciente la totalidad de los datos accesibles.

Con estos principios pedagógicos, en la experiencia hemos buscado que hacia la finalización del cursado, los alumnos puedan reconocer inductiva y deductivamente las patologías a partir de las cualidades clínicas anatomo- patológicas de los tejidos y células. El logro de esta posibilidad a nivel cognitivo, significaría una re estructuración en la estructura de pensamiento sobre el campo de la morfología celular, que mediante asimilación y acomodación del pensamiento aplicada al campo de la clínica histopatológica, pueda operar inductiva y deductivamente por medio de conceptos claros y precisos, aplicados al diagnóstico y tratamiento de las diversas dermatopatologías clínicas.
A los fines de corroborar este nivel de comprensión, hacia la finalización del ciclo lectivo, se toma una evaluación de "reconocimiento" (el proceso de reconocimiento también es un proceso cognitivo en la medida que desde el recurso iconográfico, el estudiante de medicina debe comprender los indicios de la figura en base a los conocimientos previos asimilados. De hecho, luego, en su práctica médica, los indicios que manifiestan los tejidos dermatopatológicos, aplicados a la comprensión del cuadro clínico de un paciente, le permitirá razonar sobre la patología que manifiesta el paciente) de fotografías proyectadas sobre las cuales los cursantes deben definir, en una evaluación con interrogantes de opción múltiple, la clase de tejidos y la patología que los afectan según los indicadores de la imagen. La prueba, individual, no es desarrollo, sino un test con ítems predeterminados cuyos interrogantes cuestionaban sobre la asociación entre la imagen proyectada y: el tipo de células, tipo de enfermedad elemental que muestran los tejidos, tipo de lepra, sífilis y tumores que muestran los tejidos o tipo de lesión de elemental o eritoematosas.

Los resultados y conclusiones elaborados en base a la evaluación de los alumnos de cuarto año de la Carrera de Medicina en la Cátedra de Dermatología, son aquellos se han obtenido interpretando en qué medida el "reconocimiento" - selección del ítem correcto en la evaluación de opción múltiple- de los conceptos propios de la anatomía patológica, estaban vinculados a la comprensión profunda de lo que el concepto significa y su indicador gráfico.

Los datos y el análisis de los mismos, es de tipo estadístico para hipotetizar en las discusiones, sus interpretaciones.

\section{RESULTADOS}

Los resultados demostraron algunos hechos que nos orientan a repensar tanto en la propuesta metodológica de enseñanza sostenida por la Cátedra, como el nivel de aprendizajes logrados por los alumnos a lo largo de la carrera y el nivel de razonamiento clínico que pueden lograr los estudiantes de Medicina, habiendo cursado cuatro años del grado.

Tras la administración de la evaluación sobre el nivel de significación que tienen los contenidos desarrollados en Clínica Dermatológica, se encontraron los siguientes resultados:

Los alumnos reconocieron los "tipos de células" en un $91 \%$ de manera correcta. También, con un alto indicador estadístico, reconocieron en un $87 \%$ de manera correc- 
ta, el "tipo de alteración histológica", que en la misma, correspondía a una hiperqueratosis. El mismo porcentaje de alumnos contestó de manera correcta cuando en las proyecciones se mostraron "tipos de Lesiones Elementales", conceptual y visualmente, el tipo se correspondía a "ampollas". El resto de las respuestas incorrectas, se distribuyeron en las opciones pápula y en menor medida, nódulo; mostrando en consecuencia, que los alumnos no tienen conceptos claros, precisos y distintos al respecto.

Cuando los estudiantes debieron identificar el "tipo de enfermedad dermatológica” entre las proyecciones y su asociación con los conocimientos previos, sólo el 17\% de los alumnos interpretó la fotografía de manera correcta (el tipo de correspondía con penfigoide), en tanto que el resto de los cursantes confundieron en una gran mayoría a los indicadores de la imagen con una tiña (el 27\%) y en menor medida con excemas o alopecia (el $12 \%$ y el $15 \%$, respectivamente). Asimismo, cuando la imagen proyectaba otro tipo de patología morfológica en dermatología -lesiones eritoematosas-, un 78\% de los alumnos respondió de manera correcta, siendo el tipo específico que mostraba la imagen, un caso de soriasis. El resto de la proporción de las opciones incorrectamente señaladas por los alumnos en la evaluación, confundieron la patología con un liquen, lupus o algunos señalaron que el caso no correspondía a un tipo de lesión eritoescamosa.

En el caso de "las lesiones tumorales", sólo el 2\% de los alumnos interpretó de manera correcta la imagen la cual se correspondía a un Lifoma cutáneo de células T. El resto confundió este tumor con el melanoma (el 76\%), y el resto de las opciones incorrectas con un carcinoma basocelular o espinocelular.

Finalmente, a la hora de interpretar significativamente la relación entre la fotografía de los tejidos afectados de sífilis como por lepra, los alumnos respondieron de manera correcta en un $65 \%$ respecto a la lepra, cuya variedad es la tuberculoide; en tanto que para la variedad de sífilis proyectada, el $95 \%$ demostró que sus conocimientos eran óptimos para interpretar los datos de la realidad a través de las fotografías, pues eligieron la variedad chancro de forma correcta, tal como lo ofrecía la fotografía.

\section{DISCUSIÓN}

Como se demuestra a través de la experiencia, los alumnos pueden reconocer de manera significativa los datos que ofrecen las proyecciones de casos a través de imágenes de tejidos afectados por patologías dermatológicas, dando prioridad a la comprensión de la unidad mínima de análisis en Morfología Humana: células y tejidos afectados, más que a cuadros clínicos completos; a saber, las enfermedades que pueden afectar la morfología de piel, sean: dermatológicas, elementales, eritoematosas, tumorales, lepra o sífilis. En consecuencia, en el nivel de aprendizaje de los alumnos, los niveles de comprensión significativa de los aspectos teóricos que propios de la morfología humana -en dermatología-, aún no tienen los niveles de generalidad e inclusividad que requeriría un razonamiento clínico completo que relacione inductiva y deductivamente los conceptos abordados en el estudio de la asignatura desde los niveles más concretos -las manifestaciones clínicas a través de indicadores de imágenes de los tejidos, normales o enfermos-, a otros más complejos y abstractos - las patologías en sus variedades-, lo cual requiere haber aprendido las diversas manifestaciones anatómicas de la histología y fisiopatología celular en piel.

El trabajo demuestra que una propuesta de enseñanza y aprendizaje, orientada por un enfoque cognitivo que busque la significatividad en las Ciencias Morfológicas (Piñango, 1998), debería desarrollarse con estrategias específicas de presentación y transmisión de los contenidos de la asignatura en una lógica de menor a mayor nivel de complejidad; sino también, debería poder combinarse con otras estrategias didácticas que se orienten a:

- Definir un modelo de enseñanza y aprendizaje significativo que induzca de manera permanente a la inclusión y relación de los aspectos teóricos de las ciencias morfológicas a los fines de que actúen como estructura de pensamiento que pueda incorporar datos de las manifestaciones clínicas de los tejidos normales y patológicos.

- Comprender la anatomía e histología celular en el contexto de salud y enfermedad. Analizar y relacionar la histofisiopatología en el contexto de salud del paciente en el cual se manifiesta.

- El desarrollo permanente del método científico aplicado al razonamiento clínico que avance desde los indicios hacia la comprensión del caso de manera completa.

- Entrenar a los alumnos para observar y reconocer el objeto de estudio morfológico en sus dimensiones distintas dimensiones.

- Desarrollar clases prácticas cuyo eje no sean "sólo" el dominio de habilidades y procedimientos o tecnología aplicada a los análisis de laboratorio; sino que éstos sirvan para identificar las características los tejidos, precisando: estructura, porciones y detalles más significativos como parte del sistema morfológico, propio del estudio del área. 
- Comparar según patrones topográficos, morfométricos, anatómicos e histológicos, las características del objeto de estudio, estableciendo semejanzas y diferencias para lograr una conceptualización de manera más significativa que incluya los datos empíricos de los casos y sus definiciones.
- Sostener propuestas metodológicas en la enseñanza y aprendizaje comprensivo como modelo pedagógico en la carrera, a los fines de sostener una formación basada no sólo en los razonamientos sino en la capacidad de generar acciones y prácticas profesionales médicas apoyadas en la significación.

DIONISIO DE CABALIER, M. E. \& CHALUB, D. M. The significant learning of morphological medicine sciences: experience and contributions for its education in dermatological clinic. Int. J. Morphol., 27(2):565-569, 2009.

SUMMARY: The present paper,tell us about an ealuation of students capacity in significatively recognizing,by precise and acute concepts,different manifestations of cell histopathologies in dermatology.Such a knowledge,needs a student with knowing capacity to give "meaning"to the topics wich explain the clinical manifestations-as medical reasoning way-making relationships between teached and learned knowledge.This knowing position of "significative learning"was able to be constated in the used evaluation mechanisms. In this case, is showed that students do not obtain cognitive reconstructions including in minor and mayor levels of inclusivity, the notions of cells,tissues, and pathologies showed at the images.

KEY WORDS: Significant learning; Sense; Understanding; Clinical reasoning; Recognition; Dermatologic physiohistopathology.

\section{REFERENCIAS BIBLIOGRÁFICAS}

Ausubel, D.; Novak, J. \& Hanesian, H. Psicología Educativa. Un punto de vista cognoscitivo. $2^{\mathrm{a}}$ edición. México D. F., Editorial Trillas, 2000.

Jones, B. F.; Palincsar, A. S.; Ogle, D. S.; Carr, E. G.; Wald, M. \& Marín, M. Estrategias para enseñar a aprender: un enfoque cognitivo para todas la áreas y niveles. Buenos Aires, Aique, 2001.

Lafuente, J. V.; Ruiz de Gauna, P. \& Solano, D. Enseñanza y Medicina. Mosaico de de reflexiones en torno a la docencia de las ciencias médicas. Bilbao, Servicio Editorial de la universidad del País Vasco, 1999.

Pérez, M. R. \& Díez, L. E. Diseños curriculares de aula. Un modelo de planificación como aprendizaje. Buenos Aires, Novedades Educativas, 2001.

Piñango, R. Ciencias morfológicas: un prerrequisito indiscutible para la anatomía patológica. Visión Morfológica, l(1):11, 1998.

Rosell Puig, W.; Dovale Borjas, C. \& González Fano, B. La enseñanza de las Ciencias Morfológicas mediante la integración interdisciplinaria. Educ. Med. Super., 18(1):Enero-Marzo, 2004.

Zabala, V. A. La práctica educativa. Cómo enseñar. Barcelona, Graó, 2003.
Dirección para correspondencia:

Prof. Dra. María Elisa Dionisio de Cabalier

Facultad de Ciencias Médicas.

Secretaría de Graduados U.N.C

Córdoba

ARGENTINA

Recibido : 17-06-2008

Aceptado: 31-03-2009 
